\title{
Detection and characterization of liver lesions using gadoxetic acid as a tissue-specific contrast agent
}

This article was published in the following Dove Press journal:

Biologics: Targets \& Therapy

31 July 2010

Number of times this article has been viewed

\author{
Peter Reimer' \\ Rolf Vosshenrich ${ }^{2}$ \\ 'Department of Radiology, Klinikum \\ Karlsruhe, Karlsruhe, Germany; \\ ${ }^{2}$ Radiologen-Gemeinschaftspraxis, \\ MRT im Friederikenstift, Hannover, \\ Germany
}

\begin{abstract}
The value of cross-sectional liver imaging is evaluated by the accuracy, sensitivity, and specificity of the specific imaging technique. Magnetic resonance imaging (MRI) has become a key technique for the characterization and detection of focal and diffuse liver disease. More recently, gadoxetic acid, the hepatocyte-specific MR contrast agent, was clinically approved and introduced in many countries. Gadoxetic acid may be considered a "molecular imaging" probe because the compound is actively taken into hepatocytes via the ATP-dependent organic anion transport system in the plasma membrane for the hepatic uptake. The transport of gadoxetic acid from the cytoplasm to the bile is mainly determined by the capacity of the transport protein glutathione- $S$-transferase. Gadoxetic acid enhances hepatocyte-containing lesions and improves detection of lesions devoid of normal hepatocytes, such as metastases. Innovative rapid MR acquisition techniques with near isotropic 3D pulse sequences with fat saturation parallel the technical progress made by multidetector computed tomography combined with an impressive improvement in tumor-liver contrast when used for gadoxetic acid-enhanced MRI. The purpose of this review is to provide an overview of the development, clinical testing, and applications of this novel MR contrast agent.
\end{abstract}

Keywords: hepatocytes, tumor, gadoxetic acid, liver lesions

\section{Introduction}

The clinical need for liver imaging with high accuracy, sensitivity, and specificity is a constant struggle for better imaging techniques. Gadoxetic acid may be considered a "molecular imaging" probe because the compound is actively taken into hepatocytes via ATP-dependent surface receptors. Magnetic resonance imaging (MRI) has become a key technique for the characterization and detection of focal liver disease with the use of gadoxetic acid, currently the most sophisticated cell-specific contrast agent developed for hepatic MRI. The compound enhances hepatocyte-containing lesions and improves the detection of lesions devoid of normal hepatocytes, such as metastases (Figure 1). Technical improvements of $\mathrm{T}_{1}$-weighted MRI techniques and fat-saturated 3D techniques that provide computed tomography (CT)-like impressions of anatomy further improve clinical acceptance.

The purpose of this review is to provide an overview of the development, clinical testing, and applications (Figures 1-5) of this novel MR contrast agent.

\section{Clinical background on liver tumors}

The prognosis of malignant liver tumors is, in general, poor. However, for example, $25 \%$ of patients with liver metastases from colorectal cancer may be surgical candidates, 

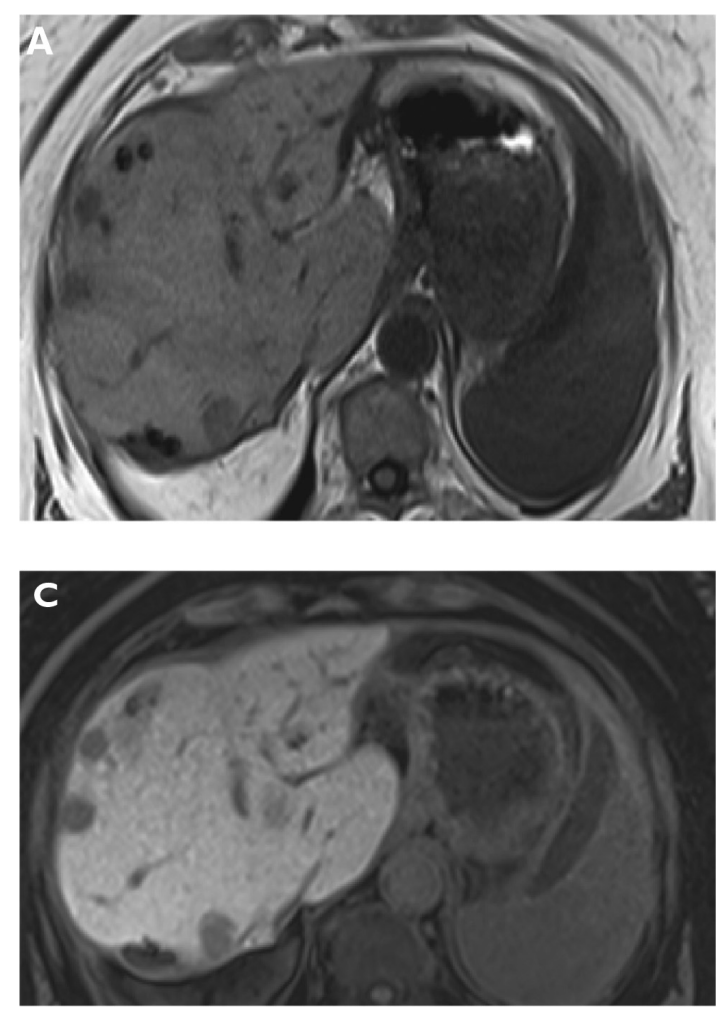
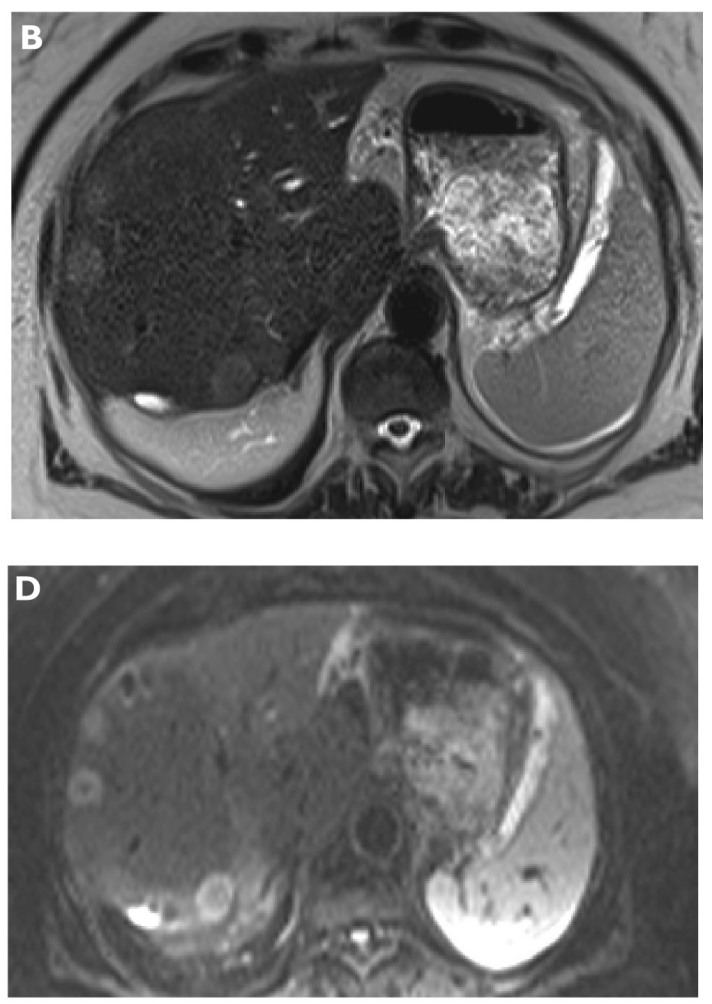

Figure I Metastases. Plain $T_{1}$-weighted spoiled 2D gradient echo (GRE) A) $T_{2}$-weighted half-Fourier acquisition single-shot turbo spin-echo (HASTE) B) hepatocellular phase gadoxetic acid-enhanced fat-suppressed (FS) $T_{1}$-weighted spoiled 3D GRE C) and diffusion-weighted imaging (DWI) D) in the axial plane. Multiple liver metastases are better visualized on gadoxetic acid-enhanced magnetic resonance imaging (MRI) and also DWI compared to plain $T_{1}$ - and $T_{2}$-weighted MRI.

with a 5-year survival of up to $40 \%$. The therapeutic armamentarium is growing significantly with the increase in the survival time of primary and secondary malignant liver tumors. ${ }^{1-3}$

Hepatocellular carcinoma (HCC) is the third leading cause of cancer mortality worldwide (Figures 3 and 4). Most of the HCCs that are associated with hepatitis B virus (HBV) occur in patients who have hepatitis B infection during most of their lives, also called chronic hepatitis B. The development of $\mathrm{HCC}$ is also associated with chronic hepatitis $\mathrm{C}$ virus (HCV). Most of the patients who develop $\mathrm{HCC}$ as a result of $\mathrm{HCV}$ will have liver cirrhosis. The average time to develop $\mathrm{HCC}$ after initial exposure to $\mathrm{HCV}$ is about 28 years and usually about 8-10 years after the development of liver cirrhosis. The way in which HCV causes HCC is not well understood. ${ }^{4}$

Cirrhosis caused by chronic alcohol consumption is the most common association of HCC in the developed world. Actually, we now understand that many of these cases are also infected with chronic HCV. The usual observation is an individual with alcoholic cirrhosis who has stopped drinking for 10 years develops HCC. It is somewhat unusual for an actively drinking alcoholic to develop HCC. When drinking is stopped, the liver cells try to heal by regenerating (reproducing). During this active regeneration, a cancerproducing genetic change (mutation) can occur, which explains the occurrence of HCC even after drinking has been stopped..$^{5-7}$ Aflatoxin B1 is the most potent liver cancercausing chemical known and has been implicated in the development of HCC in Southern China and Sub-Saharan Africa. ${ }^{8}$

Individuals with most types of cirrhosis of the liver are at an increased risk of developing HCC. In addition to the conditions described earlier (hepatitis B, hepatitis C, alcoholic cirrhosis, and hemochromatosis), $\alpha 1$-antitrypsin deficiency, a hereditary condition that can cause emphysema and cirrhosis, may lead to HCC. Liver cancer is also strongly associated with hereditary tyrosinemia, a childhood biochemical abnormality that results in early cirrhosis. Certain causes of cirrhosis are less frequently associated with HCC than are other causes. For example, HCC is rarely seen with the cirrhosis in Wilson's disease (abnormal copper metabolism) or primary sclerosing cholangitis (chronic scarring and narrowing of the bile ducts). HCC was earlier thought to be rarely found in primary biliary cirrhosis (PBC) as well. Recent studies, however, show that the frequency of HCC in PBC is comparable to that in other forms of cirrhosis. ${ }^{9}$ 

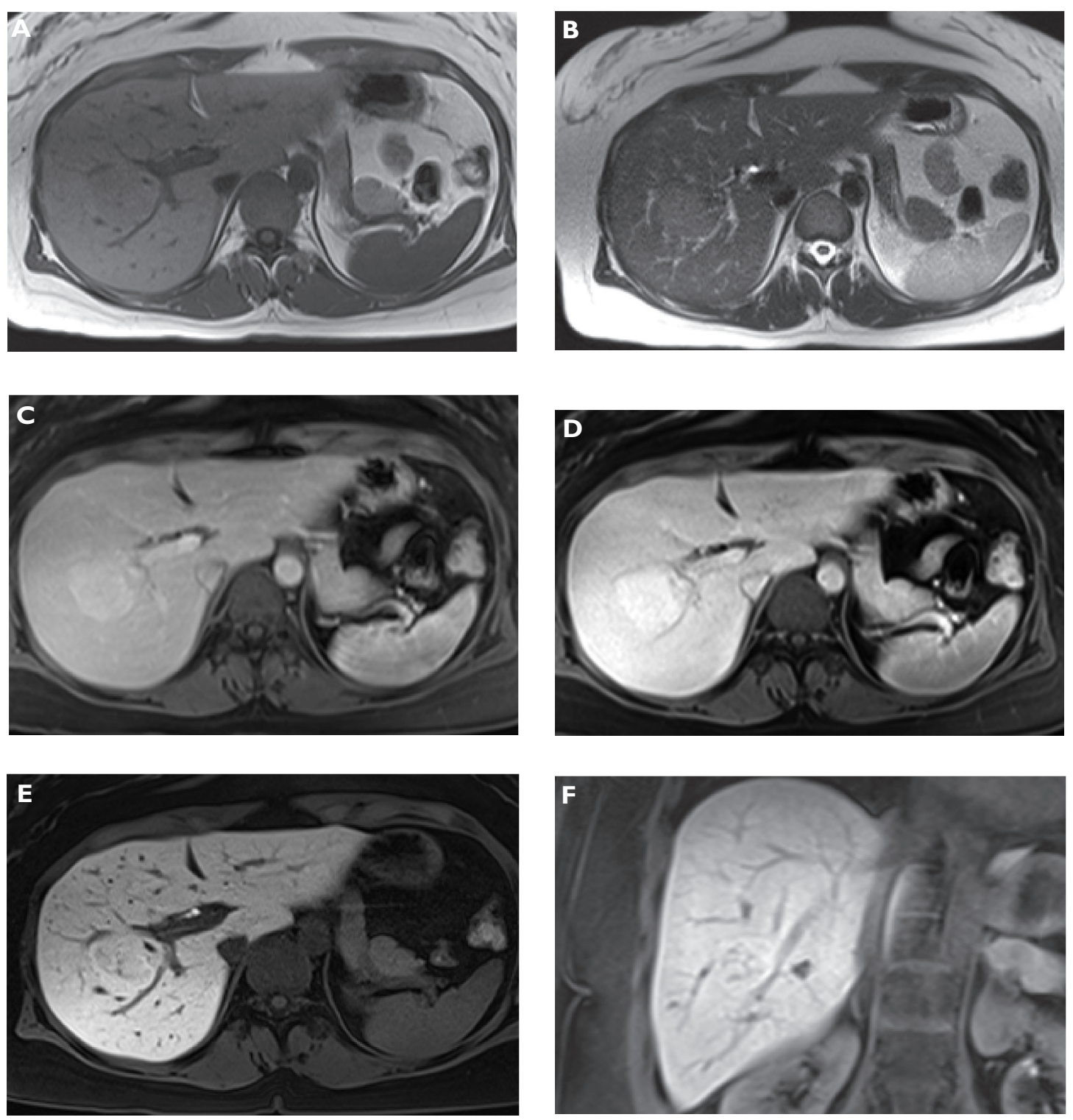

Figure 2 Focal nodular hyperplasia (FNH). Plain $T_{1}$-weighted spoiled 2D gradient echo (GRE) $\mathbf{A}_{\text {) }} \mathrm{T}_{2}$-weighted half-Fourier acquisition single-shot turbo spin-echo (HASTE) B) arterial-phase gadoxetic acid-enhanced fat-suppressed (FS) $T_{1}$-weighted spoiled 3D GRE C) portal venous-phase gadoxetic acid-enhanced FS $T_{1}$-weighted spoiled 3D GRE D) hepatocellular phase in the axial E) and coronal plane F) gadoxetic acid-enhanced FS T,-weighted spoiled 3D GRE 20 min following contrast injection. The liver demonstrates a lesion in the right liver lobe, which is slightly hypointense compared with liver on $\mathrm{T}_{1}$-weighted $\mathbf{A}$ ) and hyperintense compared on $\mathrm{T}_{2}$-weighted imaging $\mathbf{B}$ ). The tumor enhances on dynamic images (C and $\mathbf{D})$ and signal intensity stays high on late phase images due to hepatocellular uptake (E and $\mathbf{F})$ characterizing the lesion as FNH.

The prognosis of HCC is determined by the anatomic extent of tumor (stage), such as the size of the biggest lesion, number of lesions, lobar vs bilobar involvement, vascular invasion, and metastasis. ${ }^{10}$ There are other factors that are equally important: the grade or aggressiveness of the tumor. In addition to pathology, very high $\alpha$-fetoprotein is often indicative of tumors that are very aggressive. More recently, there has been very exciting work done with gene array technology to analyze the mutation rate of tumors. In HCC prognosis, the severity of cirrhosis is another very important factor, as is the functional status of the patient. Finally, the treatment administered is also important. The staging system needs to be appropriate for the treatments used. ${ }^{11,12}$

Benign liver tumors are relatively common. ${ }^{13}$ Most are asymptomatic and are detected incidentally on ultrasonography, CT, or MRI. The three most common types of benign liver tumors are hemangioma, focal nodular hyperplasia (FNH), and hepatocellular adenoma. Hemangioma is a mass of abnormal blood vessels. Up to 5\% of adults have small asymptomatic liver hemangiomas, which do not require any treatment. Sometimes, infants with large liver hemangiomas require surgery to prevent clotting. FNH is characterized by a stellate central scar and hyperplastic nodules (Figure 2). 

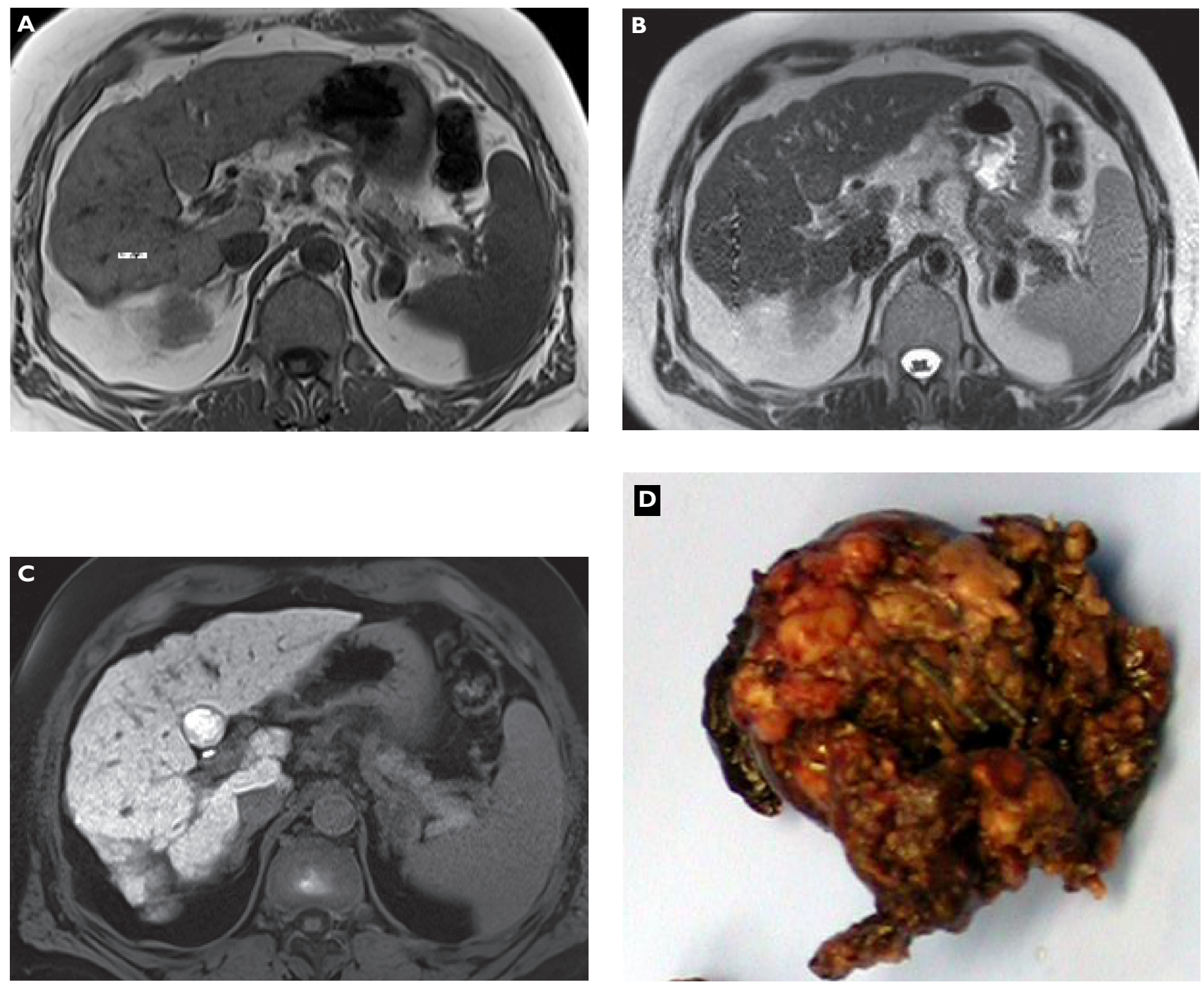

Figure 3 Well-differentiated hepatocellular carcinoma (HCC). Plain $T_{1}$-weighted spoiled 2D gradient echo (GRE) $\mathbf{A}_{\text {) }} \mathrm{T}_{2}$-weighted half-Fourier acquisition single-shot turbo spin-echo (HASTE) B) hepatocellular phase gadoxetic acid-enhanced fat-suppressed (FS) $T_{1}$-weighted spoiled 3D GRE C) and gross pathology D) in the axial plane. A small liver mass in S4b is faintly visible on unenhanced sequences showing characteristics of a HCC nodule such as a rim on $T_{1}$-weighted magnetic resonance imaging (MRI) (A). Gadoxetic acid-enhanced MRI shows an enhancing lesion mimicking a benign lesion based on uptake into the well-differentiated HCC confirmed at surgery. Gross pathology is courtesy of Prof Thomas Rüdiger.

Although some large FNHs may be associated with significant symptoms, more frequently, they are discovered incidentally by physical examination or by the development of unrelated symptoms. ${ }^{14}$ Hepatocellular adenoma occurs most often in women of childbearing age. Most of these tumors remain undetected. Sometimes, an adenoma will rupture and bleed into the abdominal cavity, requiring surgery. Adenomas rarely become cancerous. ${ }^{15}$

In the Institute for Pathology of the University of Cologne, 12,161 liver tissue cases are registered. Of them, 1,357 cases $(11.2 \%)$ showed tumors or tumor-like masses. Liver metastases of solid tumors were the largest group with 611 cases (5.0\%). Adenocarcinomas were the largest group of metastases with 400 cases (65.5\%); $48.2 \%$ of this group had metastases of colorectal cancer; $13.5 \%$, pancreatic cancer; $13 \%$, breast cancer; $6.2 \%$, gastric cancer; $4.5 \%$, lung cancer; and $3.7 \%$, esophageal cancer. ${ }^{16}$

The radiological diagnosis of liver metastasis involves detection, characterization, and tumor staging. Knowledge of the histopathologic changes that occur with metastases provides the best approach to the accurate interpretation of radiological imaging findings, and in particular, radiologists need to choose the appropriate imaging methods based on such knowledge. As most of the metastases are hypovascular, the merits of the routine acquisition of hepatic arterial dominant-phase images by contrast-enhanced MRI (CE-MRI) are disputable. Hepatic arterial dominant-phase images may be obtained when hypervascular tumors are suspected. 

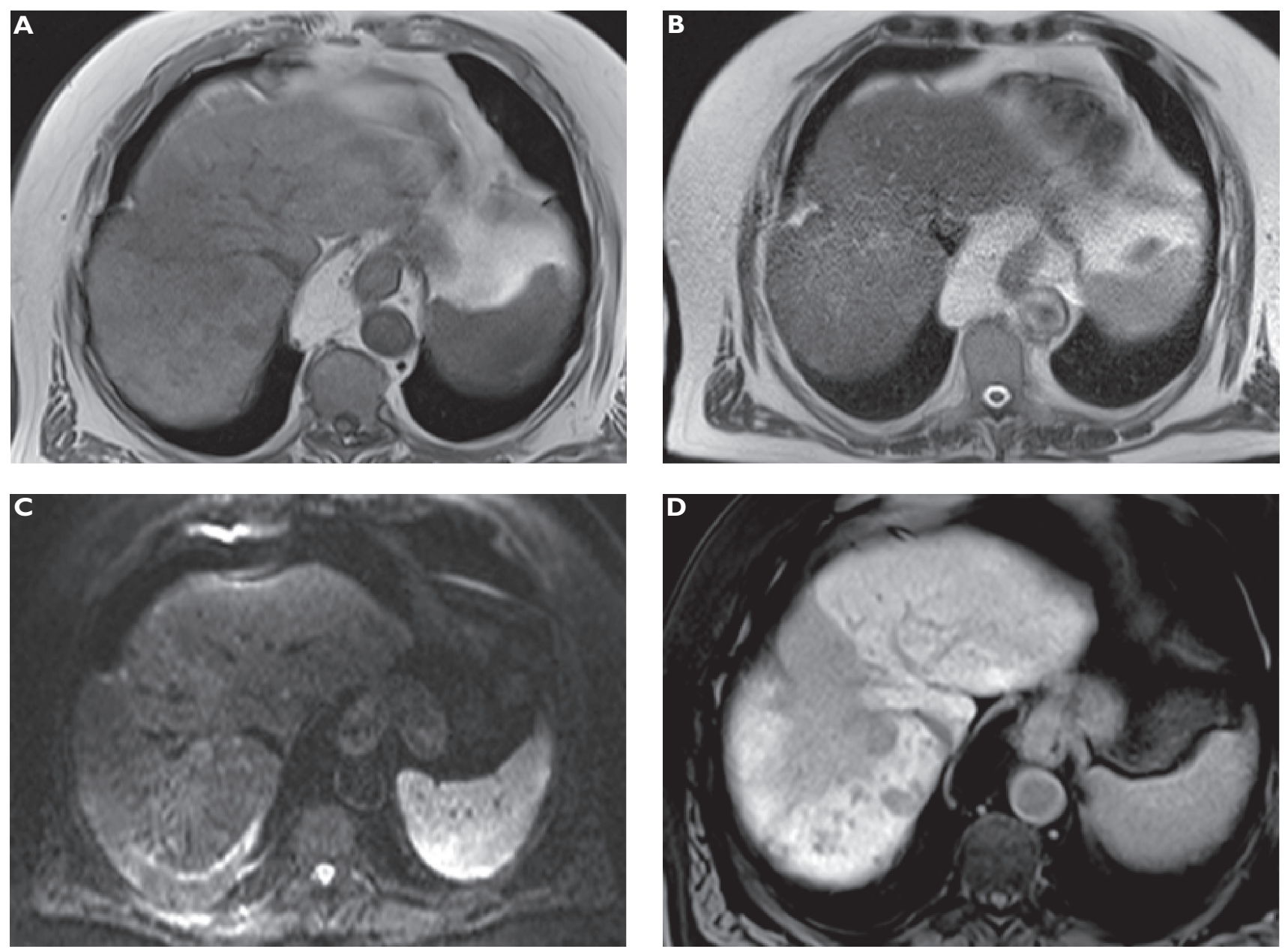

Figure 4 Anaplastic hepatocellular carcinoma (HCC) following radioembolization. Plain $T_{1}$-weighted spoiled $2 D$ gradient echo (GRE) A) $T_{2}$-weighted half-Fourier acquisition single-shot turbo spin-echo (HASTE) B) diffusion-weighted imaging (DWI) C) and hepatocellular phase gadoxetic acid-enhanced fat-suppressed (FS) $\mathrm{T}_{1}$-weighted spoiled 3D GRE D) in the axial plane. The large hypointense zone in segment 8 resembles an area following radioembolization of the previously more circumscribed tumor. Multiple new satellite tumors can only be appreciated on gadoxetic acid-enhanced MRI.

Imaging during the portal venous phase is essential for detecting metastases, evaluating intrahepatic vessel invasion, and assessing intratumoral necrosis or fibrosis. Equilibrium- to delayed-phase imaging performed 3-5 minutes after the administration of contrast agent may improve the detection of intratumoral fibrosis and may occasionally lead to more accurate tissue characterization. MRI offers diagnostic information on vascularity, amount of free water, hemorrhage, fibrosis, necrosis, and water molecule diffusion in metastases. ${ }^{17}$

\section{Rationale for gadoxetic acid-enhanced MRI}

Gadoxetic acid was developed to improve the detection and characterization of focal liver disease (Figures 1-5) compared with nonspecific low-molecular weight gadolinium chelates. ${ }^{18-21}$ At the time of development, dynamic
$\mathrm{T}_{1}$-weighted MRI was not able to cover the entire liver during bolus injection of nonspecific low-molecular weight gadolinium chelates comparable to CT. Therefore, it was hypothesized that an agent that may stay in the liver for some time may allow for better imaging at a higher resolution. ${ }^{22}$ The gadopentetate dimeglumine molecule serves as the backbone with an added lipophilic side chain binding to the surface receptors on the hepatocytes. Up to $50 \%$ of the agent is subsequently taken up into hepatocytes and later released into the bile. ${ }^{18}$ Agents with these characteristics are called hepatocyte-specific or hepatobiliary contrast agents, which have a hepatocellular phase during which the contrast agent is concentrated in hepatocytes before being released into the bile. This phase may last for several hours, and the contrast agent in the hepatocytes shortens $\mathrm{T}_{1}$-relaxation time, thus significantly increasing the signal on $\mathrm{T}_{1}$-weighted images. It has been shown that gadoxetic acid enters plasma mem- 

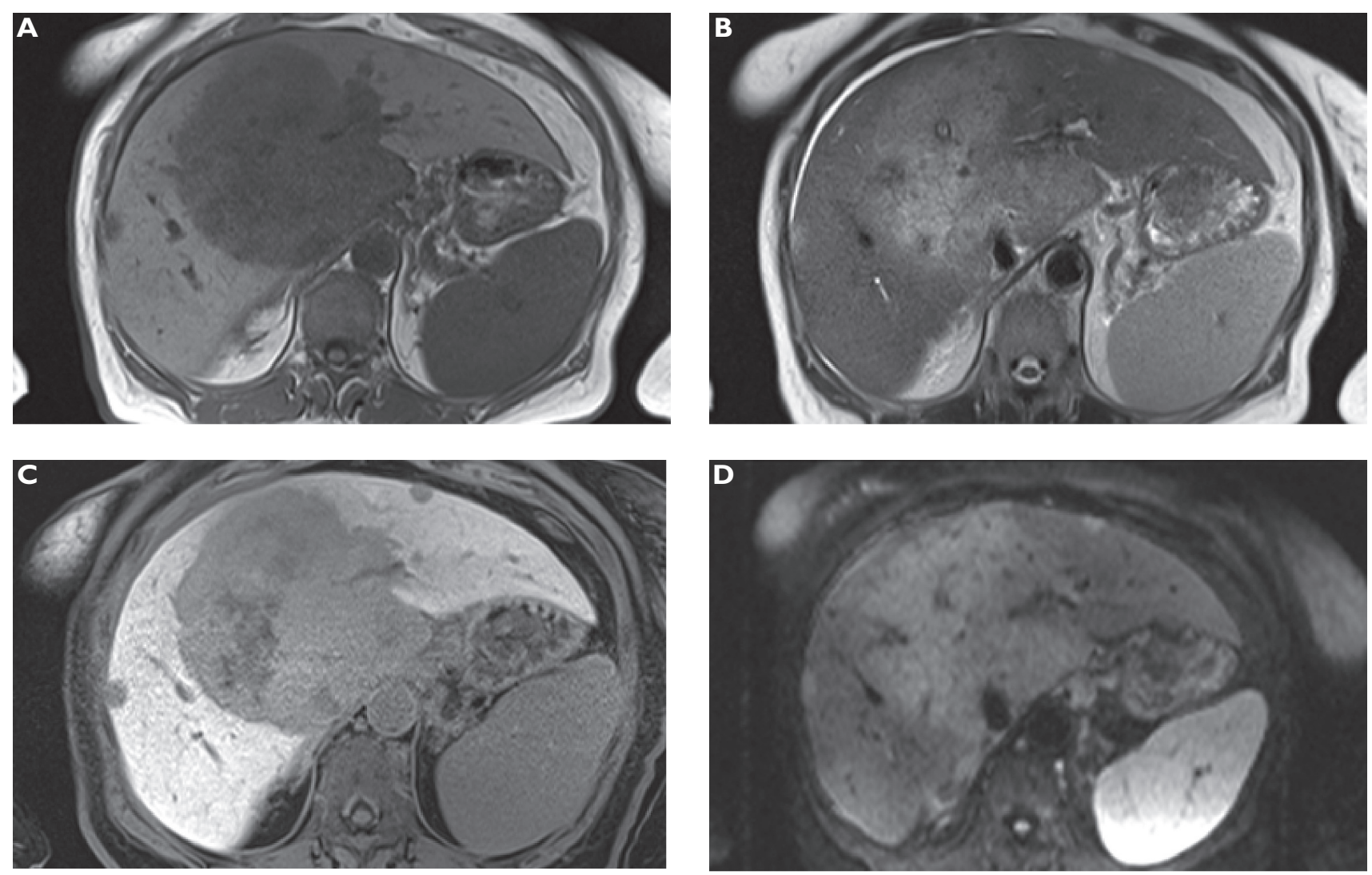

Figure 5 Cholangiocellular carcinoma. Plain $T_{1}$-weighted spoiled 2D gradient echo (GRE) A) $T_{2}$-weighted half-Fourier acquisition single-shot turbo spin-echo (HASTE) B) hepatocellular phase gadoxetic acid-enhanced fat-suppressed (FS) $T_{1}$-weighted spoiled 3D GRE C) and diffusion-weighted imaging (DWI) D) in the axial plane. A large central liver mass is demonstrated on unenhanced sequences. Gadoxetic acid-enhanced magnetic resonance imaging (MRI) shows the lesion much better and additional satellite lesions in both liver lobes with increased conspicuity.

brane vesicles following a linear, concentration-dependent mechanism up to $1.5 \mathrm{mM}$ of substrate. Sulfobromophthalein and taurocholate, but not unconjugated bilirubin, inhibited the rate of uptake. ${ }^{23}$ The pharmacokinetics of gadoxetic acid is characterized by a capacity-limited transport process via the biliary route of elimination, thus strongly resembling the pharmacokinetics of some biliary x-ray contrast media (iotroxic, iodipamic, or idoxamic acid) or the synthetic dyes (indocyanine green). ${ }^{24}$ Michaelis-Menten kinetics in the extrarenal elimination was considered as the rate-limiting process of gadoxetic acid, the binding to plasma protein of which is small $(10.3 \pm 1.4 \%)$. Biliary elimination was significantly inhibited by the intravenous (IV) coadministration of sulfobromophthalein, whereas tauroglycocholate revealed no effect, indicating the involvement of the socalled organic anion plasma membrane transport system for the hepatic uptake. The transport of gadoxetic acid from the cytoplasm to the bile is mainly determined by the capacity of the transport protein glutathione- $S$-transferase, as demonstrated by in vitro binding studies. A hepatobiliary transport maximum of $9.2 \mu \mathrm{mol} / \mathrm{min} \cdot \mathrm{kg}$ was evaluated by infusion studies. No metabolites were detected either in the bile or in the urine, and enterohepatic circulation can be excluded. ${ }^{25,26}$

\section{Pharmacology of gadoxetic acid}

Gadolinium-ethoxybenzyl-diethylenetriamine-pentaaceticacid (Gd-EOB-DTPA, SH L 569 B $^{\circledR}$; gadoxetic acid disodium, Primovist ${ }^{\circledR}$ outside the United States or Eovist ${ }^{\circledR}$ in the United States) is a paramagnetic hepatobiliary contrast agent with hepatocellular uptake via the anionic-transporter protein and a molecular weight of $725.71 \mathrm{Da}\left(\mathrm{C}_{23} \mathrm{H}_{28} \mathrm{GdN}_{3} \mathrm{Na}_{2} \mathrm{O}_{11}\right){ }^{18}$ The compound was extensively tested preclinically in various species. ${ }^{24-35}$ Gadoxetic acid exhibits a $\mathrm{T}_{1}$-relaxivity of $4.9 \mathrm{mM}^{-1} \cdot \mathrm{s}^{-1}$ at $0.47 \mathrm{~T}$ in water, comparable to that of gadopentetate dimeglumine $3.7 \mathrm{mM}^{-1} \cdot \mathrm{s}^{-1}$, but shows a higher $\mathrm{T}_{1}$-relaxivity $\left(\mathrm{R} 18.2 \mathrm{mM}^{-1} \cdot \mathrm{s}^{-1}\right.$ ) in human plasma than gadopentetate dimeglumine ( $\left.\mathrm{R} 15.0 \mathrm{mM}^{-1} \cdot \mathrm{s}^{-1}\right)$. This may be explained by the greater degree in protein binding $(10.7 \% \pm 3.4 \%)$ compared with gadopentetate dimeglumine $(1.6 \% \pm 4.2 \%)$. At $37^{\circ} \mathrm{C}$, the solution has an osmolality of $0.89 \mathrm{osmol} / \mathrm{kg} \mathrm{H}_{2} \mathrm{O}$ and a viscosity of $1.22 \mathrm{mPas}$. Gadoxetic 
acid is an aqueous formulation with a concentration of $0.25 \mathrm{~mol} / \mathrm{L}$ and a thermodynamic stability constant of $10 .{ }^{20}$ Biodistribution studies in dogs using radioactive ${ }^{153}$ Gd-labeled Gd-EOB-DTPA revealed a dose-dependent renal $(40.9 \% \pm 2.35 \%)$ and biliary $(57.0 \% \pm 2.49 \%)$ excretion without signs for metabolism and an enterohepatic recirculation of approximately $2.1 \% \pm 0.56 \% .^{24-26,35}$

Gadoxetic acid was well tolerated within phase 1 trials at doses of $10,25,50$, and $100 \mu \mathrm{mol} \mathrm{Gd} / \mathrm{kg}$, with no important side effects or changes in laboratory parameters. ${ }^{27}$ Results of laboratory tests, clinical measurements, and pharmacokinetic data were obtained in 44 healthy volunteers in a double-blinded, randomized, placebo-controlled design. MR images were obtained in another 16 healthy volunteers before and after 6 hours of fast IV administration of gadoxetic acid. Homogeneous enhancement of liver parenchyma was observed immediately after injection of the contrast agent. Peak liver signal intensity (SI) was noted 20 minutes after injection, followed by plateau-like enhancement over about 2 hours. The common bile duct was hyperintense within 10 minutes after injection in all volunteers. The time course of contrast enhancement in bile ducts and the gallbladder showed intense signal enhancement beginning 5-16 minutes after injection (mean, 10 minutes) and persisting for as long as 360 minutes. The duration of signal enhancement was significantly longer for higher doses $(50,100 \mu \mathrm{mol} / \mathrm{kg})$ than for lower doses $(10,25 \mu \mathrm{mol} / \mathrm{kg})$. Intrahepatic bile ducts were hyperintense when compared with liver parenchyma in all subjects receiving $10 \mu \mathrm{mol} / \mathrm{kg}$ contrast agent from approximately 50-120 min after its application. Intrahepatic bile ducts were not displayed using the higher doses, probably because of the strong enhancement of the liver parenchyma. Gallbladder contrasting was achieved in all cases beginning 7-33 minutes after injection (mean, 19 minutes) and remained visible for up to 360 minutes in $94 \%$ of cases. Hyperintense visualization of normal extrahepatic bile ducts as well as the gallbladder was regularly achieved. ${ }^{36}$

During 2 subsequent clinical phase 2 trials, the diagnostic efficacy and safety of gadoxetic acid were explored at 5 doses $(3.0 ; 6.0 ; 12.5,25$, and $50 \mu \mathrm{mol}$ gadoxetic acid $/ \mathrm{kg}$ body weight $[\mathrm{bw}])$ when compared with placebo ( $0.9 \%$ saline) in patients with known focal liver lesions. These 2 clinical phase 2 trials revealed a lowest effective dose of $25 \mu \mathrm{mol}$ gadoxetic $\mathrm{acid} / \mathrm{kg} \mathrm{bw} .{ }^{37}$ The number of patients with more lesions being visualized on postcontrast than on precontrast scans increased with increasing dose up to $12.5 \mu \mathrm{mol}$ gadoxetic acid $/ \mathrm{kg}$ bw. The enhancement of hepatic vessels and liver parenchyma during dynamic imaging following bolus injection at $2 \mathrm{~mL} / \mathrm{s}$ also increased with increasing dose. There was no significant improvement in quantitative or qualitative efficacy parameters at 45 minutes after injection compared with 20 minutes after injection. Dynamic imaging can start immediately after bolus injection, and accumulation phase imaging can be performed at 20 minutes after injection. ${ }^{37-40}$

The subsequent European phase 3 multicenter trial was performed with a dose of $25 \mu$ mol gadoxetic $\mathrm{acid} / \mathrm{kg}$ bw (BW). ${ }^{41,42}$ The safety and efficacy of gadoxetic acid disodium-enhanced MRI for the detection of focal liver lesions were investigated with a study design correlating MRI with the results of histopathologic examination and/or intraoperative ultrasonography used as a standard of reference. In the off-site evaluation, 2 of the 3 blinded readers showed a statistically significant difference in lesion detection between precontrast and postcontrast MRI. A large number of additionally correctly detected and localized lesions were smaller than $1 \mathrm{~cm}$. No clinically relevant changes in hemodynamic or laboratory parameters were observed. No death or any adverse event (AE) leading to discontinuation of the study was reported. The most frequently reported symptoms of definitely or probably related AEs were nausea, vasodilatation, headache, taste perversion, and pain at the injection site. $^{42}$

\section{Efficacy}

A second phase 3 multicenter trial was performed in the United States between September 1998 and April 2000 to assess the efficacy and safety of postcontrast MRI with gadoxetic acid compared with that of precontrast MRI in patients who were known to have or suspected to have liver lesions and who were scheduled for hepatic surgery. A total of 172 patients were enrolled. After precontrast MRI, 169 patients (94 men, 75 women) received an IV bolus injection of $25 \mu \mathrm{mol} / \mathrm{kg}$ gadoxetic acid and underwent dynamic and delayed MRI 20 minutes after the injection. The standard of reference was surgery with intraoperative ultrasonography, biopsy, and pathologic evaluation of resected liver segments and/ or 3-month follow-up of nonresected segments. Using MRI, 316 lesions were identified in 131 patients. In 77\%, 72\%, and $71 \%$ of patients for readers 1,2 , and 3 , respectively, more lesions were observed using combined precontrast and post contrast MRI than using precontrast MRI alone. Compared with precontrast MRI, postcontrast MRI with gadoxetic acid demonstrated improved sensitivity for lesion detection in most of the blinded readers, with no substantial AEs. Sensitivity values for blinded readings were significantly greater at postcontrast MRI than at precontrast MRI. ${ }^{43}$ 
More recently, Hammerstingl et $\mathrm{al}^{41}$ published a multimember study on the diagnostic efficacy of CE-MRI with gadoxetic acid as opposed to contrast-enhanced CT in the diagnosis of focal liver lesions. A total of 169 patients with lesions were enrolled, and they underwent CE-MRI with gadoxetic acid and CT within 6 weeks. The standard of reference was established by evaluating the liver specimens of 131 patients with 302 focal lesions using intraoperative ultrasonography. The strength of detecting small focal lesions using CE-MRI with gadoxetic acid is shown by the highest rate of correctly detected lesions with a diameter below $1 \mathrm{~cm}$. Differential diagnosis was also superior for CE-MRI with gadoxetic acid (82.1\%) vs CT (71.0\%). Subsequently, a change in surgical therapy was achieved in $14.5 \%$ of patients. Therefore, CE-MRI with gadoxetic acid was superior in the diagnosis and therapeutic management of focal liver lesions compared with CT.

\section{Safety}

Gadoxetic acid is currently an US Food and Drug Administration (FDA) category $\mathrm{C}$ drug - the effect of which upon a newborn is unknown. When using gadoxetic acid, the usual safety precautions for MRI must be observed, for example, exclusion of cardiac pacemakers and ferromagnetic implants. The patient should refrain from eating for 2 hours prior to examination to reduce the risk of aspiration, as nausea and vomiting are known possible adverse reactions. The contrast agent should be administered with the patient lying down. After the injection, the patient should be kept under observation for at least 30 minutes, because most of the undesirable effects are found to occur within this time. This medicinal product contains $11.7 \mathrm{mg}$ sodium $/ \mathrm{mL}$, and the dosage is $0.1 \mathrm{~mL} /$ $\mathrm{kg}$ bw. This may be taken into consideration by patients on a controlled sodium diet.

There have been reports on nephrogenic systemic fibrosis (NSF) associated with the use of some gadolinium-containing contrast agents in patients with acute or chronic renal impairment (glomerular filtration rate $<30 \mathrm{~mL} / \mathrm{min} / 1.73 \mathrm{~m}^{2}$ ), acute renal insufficiency of any severity due to the hepatorenal syndrome, or in the perioperative liver transplantation period. As there is a possibility that NSF may occur with the use of Primovist, it should, therefore, only be used in these patients after careful risk/benefit assessment, if the diagnostic information is essential and not available with non-CE-MRI. All patients should be screened, in particular, patients older than 65 years, for renal dysfunction by obtaining a history of laboratory tests.
There is no evidence to support the initiation of hemodialysis for the prevention or treatment of NSF in patients not already undergoing hemodialysis. Caution should be exercised in patients with severe renal impairment due to the reduced elimination capacity of gadoxetic acid.

Care should be taken when gadoxetic acid is administered to patients with severe cardiovascular problems because only limited data are available so far. The contrast agent should not be used in patients with uncorrected hypokalemia. Special care should be taken in the following patient groups: patients with (1) known congenital long QT syndrome or a familial history of congenital long QT syndrome; (2) known previous arrhythmias who are currently taking class III antiarrhythmic drugs, such as amiodarone and sotalol, which prolong cardiac repolarization. Gadoxetic acid may cause transient QT prolongation in individual patients.

Allergy-like reactions, including shock, are known to be rare events after the administration of gadolinium-based MRI contrast media. Most of these reactions occur within half an hour after the administration of contrast media. However, as with other contrast media of this class, delayed reactions may occur after hours to days in rare cases. Medication for the treatment of hypersensitivity reactions and preparedness for the institution of emergency measures are necessary. The risk of hypersensitivity reactions is higher in case of previous reaction to contrast media or history of bronchial asthma or allergic disorders.

Hypersensitivity reactions can be more intense in patients on $\beta$-blockers, particularly in the presence of bronchial asthma. It is considered that patients on $\beta$-blockers might be refractory to standard treatment of hypersensitivity reactions with $\beta$-agonists. If hypersensitivity reactions occur, injection of the contrast medium must be discontinued immediately.

Intramuscular administration may cause local intolerance reactions, including focal necrosis and should therefore be strictly avoided. No interaction studies have been performed in humans. However, in general, anionic drugs primarily excreted into the bile, such as rifampicin, may interfere in the hepatic contrast enhancement and the biliary excretion of gadoxetic acid. Animal studies demonstrated that compounds belonging to the class of rifamycins block the hepatic uptake of gadoxetic acid, thus reducing the hepatic contrast effect. In this case, the expected benefit of an injection of gadoxetic acid might be limited. No further interactions with other medicinal products are known. Elevated levels of bilirubin or ferritin can reduce the hepatic contrast effect of gadoxetic acid. Serum iron determination using complexometric methods (eg, ferrocine complexation method) may result in false 
values for up to 24 hours after the examination with gadoxetic acid because of the free complexing agent contained in the contrast medium solution. ${ }^{44,45}$

In a more recent trial analyzing the efficacy and safety of gadoxetic acid in Chinese patients, no serious AEs were observed in 234 patients ( 79 female, 155 male; age, 50.2 years). Other AEs were reported in 20 of 234 patients, including nausea (2 patients), pain at the injection site (1), increased systolic/diastolic blood pressure (2), increased blood glucose (2), decreased white blood cell count (2), increased blood bilirubin (3), increased blood phosphorus (1), increased alanine aminotransferase (1), increased aspartate aminotransferase (1), hypertension (4), and hypotension (2). ${ }^{46}$

\section{Effect of hepatic and renal impairment}

The impact of hepatic and/or renal impairment was investigated in a special population study. Groups of volunteers with various levels of impaired hepatic or renal function, coexisting hepatic and renal impairment, and a control were enrolled. Pharmacokinetic parameters of gadoxetic acid derived from the serum level data were markedly altered only in end-stage renal failure. Mild to severe hepatic impairment and moderate renal impairment did not markedly change the pharmacokinetic parameter values. Considering the safety profile, the observed differences in the patients with end-stage renal failure may not be clinically relevant. Hepatic parenchymal enhancement with a standard dose of gadoxetic acid was accomplished in all patient groups. For clinical use, the enhancement was sufficient above $50 \%$, and of sufficient duration with 60 minutes or more. No special considerations appear to be required in the presence of end organ disease. Gadoxetic acid, as a single rapid IV dose of $25 \mu \mathrm{mol} / \mathrm{kg}$ bw, was safe and well tolerated by all patients in this study. This included patients who had hepatic or renal impairment (including end-stage renal disease), patients with both hepatic and renal impairment, and healthy male and female volunteers (younger and older than 65 years) with normal liver and kidney function. ${ }^{47}$

\section{Patients}

Gadoxetic acid is generally well tolerated by patients in clinical trials and clinical practice as observed in a most recent trial. ${ }^{46}$ Most undesirable effects were transient and of mild to moderate intensity. The most commonly noted AEs were nausea and headache with an incidence of $1.1 \%$. Coldness, warmth, or pain at the injection site, injection site reaction, and accumulation of fluid at the injection site were rare. The clinical usefulness and accuracy have already been covered beyond reports on clinical trials. Zech et $\mathrm{al}^{48,49}$ presented an economic evaluation comparing gadoxetic acid with extracellular contrast-media-enhanced MRI (ECCMMRI) and 3-phase multidetector computed tomography (MDCT) in the development of patients with metachronous colorectal liver metastases. MRI with gadoxetic acid potentially leads to cost savings by improving preoperative planning and decreasing intraoperative changes. ${ }^{42}$

\section{Clinical applications Liver}

Liver imaging with gadoxetic acid typically consists of the acquisition of precontrast $\mathrm{T}_{1}$ - and $\mathrm{T}_{2}$-weighted images followed by bolus injection and dynamic $\mathrm{T}_{1}$-weighted imaging of the perfusion phase. Since the injected dose is just one quarter of the regular gadolinium dose, contrast enhancement is weaker than that experienced with nonspecific gadolinium chelates. ${ }^{50}$ Nevertheless, the perfusion phase allows for some assessment of perfusion patterns of liver lesions. Accumulation-phase images may be obtained upon washout of unspecifically perfused liver lesions and may start as early as 8 minutes after injection as tested in phase 3 trials. ${ }^{42}$ Acquiring $\mathrm{T}_{2}$-weighted images following perfusion-phase imaging shortens the examination time with reproducible image quality. ${ }^{51}$ Gadoxetic acid improves the detection of a spectrum of liver lesions, especially smaller lesions. These results apply mainly to the accumulation-phase images, while perfusionphase images are more utilized for lesion characterization. For characterization, the per-patient sensitivity was significantly higher on postcontrast images alone or on combined images compared with the precontrast MR images. ${ }^{42}$

\section{Metastases}

Tumors not derived from hepatocytes, such as liver metastases or cholangiocarcinoma do not take up the gadoxetic acid in the accumulation phase, thereby increasing the postcontrast tumor conspicuity. During the perfusion phase, liver metastases show the highest enhancement of 90-120 seconds following IV injection of gadoxetic acid. Tumor enhancement then gradually decreases and stabilizes after 10 minutes. Liver enhancement is stronger than the enhancement of metastases after 3 minutes following IV injection of gadoxetic acid. Therefore, lesion-liver contrast of liver metastases gradually increases from 2 minutes postcontrast to 10 minutes postcontrast. The increase from precontrast and early postcontrast (2 minutes) SIs to later postcontrast SIs ( $\geq 10$ minutes) is 
significant; however, differences among later postcontrast time points $\left(10,20\right.$, and 45 minutes) are not significant. ${ }^{52}$ Gadoxetic acid provides improved detection and characterization of liver metastases compared with unenhanced and Gd-enhanced MRI, both experimentally and clinically. Almost no metastasis showed complete enhancement during dynamic imaging as investigated during the US phase 3 trial. Moreover, in some cases, there is partial, often rim enhancement in metastases, which do not possess a uniform and homogeneously distributed vasculature. In the accumulation phase, most metastases do not enhance (Figure 1). ${ }^{42}$

\section{Hepatocellular carcinoma}

Depending on the tumor vascularity, some tumors may enhance stronger and some may enhance weaker than normal liver parenchyma as also known for gadolinium chelates (Figures 3 and 4). ${ }^{53,54}$ Gadoxetic acid has the potential capacity for the concurrent assessment of vascularity of HCC and hepatocellular-specific properties within the tumor. ${ }^{55}$ Persisting enhancement during the accumulation phase has been subject to experimental and clinical reports. ${ }^{56}$ Fujita et $\mathrm{al}^{57}$ described potential enhancement of moderately differentiated HCCs in a mice model. In the US phase 3 trial, HCCs were usually described as enhancing heterogeneously in the dynamic phase. Enhancement was greater during the dynamic phases than during the hepatocyte phase..$^{43}$ In a subgroup analysis within the European phase 3 trial, 40 patients with histopathologically proven $\mathrm{HCC}$ were selected. No difference was found between combined pre- or postcontrast MRI and spiral CT for lesion classification. ${ }^{58}$ Considering typical enhancement patterns, gadoxetic acid-enhanced MRI during the accumulation phase helps discriminating $\mathrm{HCC}$ from arterial-enhancing pseudolesions during the arterial phase. In a recent study by Sun et al, ${ }^{59} 42$ of 44 HCCs demonstrated low SI and only 2 showed iso or high SI on the hepatobiliary phase of gadoxetic acid-enhanced MRI. Alternatively, 50 of 53 arterial-enhancing pseudolesions showed iso SI on the hepatobiliary phase, and only 2 showed low SI.

Narita et $\mathrm{a}^{60}$ investigated the uptake of gadoxetic acid in the hepatobiliary phase in HCC. Enhancement ratios (ERs) and expression levels of the organic anion transporter (OATP) $1 \mathrm{~B} 3$ protein were examined. Gadoxetic acid accumulated in the hepatobiliary phase in 6 of the 22 cases. All 6 gadoxetic acid-positive cases were moderately differentiated HCC, but 11 other moderately differentiated HCCs did not show gadoxetic acid uptake. Histopathologically, 4 gadoxetic acidpositive HCCs and 5 gadoxetic acid-negative HCCs produced bile. HCCs with gadoxetic acid uptake overexpressed OATP1B3 compared with HCCs without gadoxetic acid uptake, and OATP1B3 levels were significantly correlated with ERs ( $r=0.91, P<0.0001)$. The study elegantly revealed that uptake of gadoxetic acid in HCC is determined by the expression of OATP1B3 rather than by tumor differentiation or bile production. This potential problem is underestimated in most studies. ${ }^{61,62}$

\section{Hemangioma}

Enhancement of liver hemangioma is typically visible during the perfusion phase up to 10 minutes following IV injection of gadoxetic acid. ${ }^{40,63}$ In the US phase 3 trials, an increasing proportion of hemangiomas displayed partial enhancement as time elapsed in the dynamic phase. In the accumulation phase, most of the hemangiomas did not enhance, or only partially enhanced. ${ }^{43}$ Delayed images obtained at 45 minutes showed no significant difference of hemangiomas compared with metastases or HCCs. Hemangiomas initially demonstrated a slight decrease in lesion-liver contrast due to the relative tumor enhancement following IV injection of gadoxetic acid. The increase from precontrast to early postcontrast values up to 10 minutes was not significant, and lesion-liver contrast still increased between 20 and 45 minutes $(P \leq 0.05) .{ }^{52}$ Liver hemangiomas are more conspicuous on turbo spin echo (TSE)- $\mathrm{T}_{2}$ images than on gradient echo (GRE)- $\mathrm{T}_{1}$ or short-tau inversion recovery (STIR) images following gadoxetic acid administration, and the conspicuity on TSE- $T_{2}$ images is not affected by the contrast agent. ${ }^{64}$

\section{$\mathrm{FNH}$ and adenoma}

Hepatocyte-derived benign liver tumors exhibit prolonged tumor enhancement due to specific intracellular uptake of gadoxetic acid (Figure 2). Most of FNH lesions enhance completely during dynamic-phase imaging and accumulation-phase imaging, most FNH show either complete or partial enhancement. ${ }^{43}$ Zech et al ${ }^{65}$ looked at the diagnostic performance of MRI with gadoxetic acid in comparison with precontrast MRI and CT in the specific diagnosis FNH. Characterization and enhancement patterns were evaluated in 59 confirmed FNH lesions. Complete or partial enhancement was present in the early dynamic phase in most lesions (80\%-90\%) and was mainly homogenous. Enhancement in the hepatocyte phase after 10 and 20 minutes was observed in up to $90 \%$ of lesions. FNH lesions show similar enhancement characteristics to those of extracellular contrast agents in the early dynamic phase 
after bolus injection. During the hepatocyte phase, after 20 minutes, enhancement is regularly seen. ${ }^{66}$

Adenomas display considerable variability in enhancement, during both dynamic-phase and accumulation-phase imaging. Some lesions show homogeneous enhancement to the same degree as, or even more than, the surrounding parenchyma. The lack of a $\mathrm{T}_{2}$-weighted hyperintense central scar is important to avoid a misdiagnosis of FNH. Some adenomas show only minimal uptake, which does not allow reliable differentiation between adenoma and well-differentiated HCC so far. In one series of images, intracellular uptake of gadoxetic acid in adenomas was found to be absent or strongly reduced during the hepatocyte phase. Therefore, the differentiation of adenomas from dysplastic or malignant lesions is not possible. ${ }^{49,53,63,67}$

\section{Biliary system}

Imaging of biliary excretion and hepatic function with gadoxetic acid-enhanced MRI has been investigated experimentally in normal and diseased liver. ${ }^{68-71}$ Due to the strong biliary excretion of gadoxetic acid in humans, the enhancement of the biliary system and drainage into the duodenum are visualized within several minutes after administration in patients without biliary obstruction. ${ }^{72}$ Analysis of the course of enhancement in bile ducts and the gallbladder revealed that the common bile duct showed dose-dependent intense signal enhancement beginning at less than 5 minutes after injection and persisting for more than 2 hours. Intrahepatic bile ducts are hyperintense when compared with liver parenchyma. The gallbladder shows signal enhancement typically beginning at $>7$ minutes after injection and remains visible for up to 6 hours in most subjects. ${ }^{36}$ Residual hepatic enhancement during the accumulation phase does interfere with biliary visualization. ${ }^{73,74}$ The added value of gadoxetic acid-enhanced magnetic resonance cholangiography $(\mathrm{MRC})$ to unenhanced magnetic resonance cholangiopancreatography (MRCP) is considered modest, and the indication should be limited to selected cases. ${ }^{73,74}$

\section{Comparative imaging studies}

There are several comparative studies available, which may show the value of gadoxetic acid from a more practical perspective. A randomized study comparing liver enhancement with gadoxetic acid and gadobenate dimeglumine in 295 patients demonstrated that liver enhancement in the accumulation phase after the injection of gadoxetic acid was superior to that of gadobenate dimeglumine. ${ }^{75}$

Hammerstingl et $\mathrm{al}^{41}$ published a multicentric trial comparing MRI with gadoxetic acid and CT in the diagnosis of focal liver lesions with a standard of reference of 169 patients with hepatic lesions eligible for surgery. In the blinded reading, there was a trend toward gadoxetic acid-enhanced MRI, not reaching statistical significance. The highest rate of correctly detected lesions with a diameter below $1 \mathrm{~cm}$ was achieved by gadoxetic acid-enhanced MRI. Differential diagnosis was superior for gadoxetic acid-enhanced MRI with $82.1 \%$ vs CT with $71.0 \%$. A change in surgical therapy was documented in 19 of 131 patients (14.5\%) who underwent postgadoxetic acid-enhanced MRI. Gadoxetic acid-enhanced MRI was considered superior in the diagnosis and therapeutic management of focal liver lesions compared with $\mathrm{CT}^{4}{ }^{42}$

Ichikawa et $\mathrm{al}^{76}$ studied and compared gadoxetic acidenhanced MRI with unenhanced MRI and triphasic CT for the detection and characterization of focal liver lesions in 178 patients with suspected focal hepatic lesions enrolled in a multimember trial. Gadoxetic acid-enhanced MRI improved the detection and characterization of focal hepatic lesions compared with unenhanced MRI. Combined MRI showed higher sensitivity in lesion detection than CT, with the results being statistically significant for on-site readers and 2 of 3 blinded readers. Higher sensitivity in lesion detection using combined MRI compared with CT was also clearly demonstrated in the following subgroups: (1) patients with lesions with a diameter $\leq 20 \mathrm{~mm}$; (2) patients with cirrhosis; and (3) patients with HCC.

Kim et $\mathrm{al}^{62}$ compared the diagnostic performance of gadoxetic acid-enhanced MRI at 3 Tesla with that of triple-phase 16-, 40-, and 64-MDCT in the preoperative detection of HCC in 62 consecutive patients. The diagnosis of HCC was established after surgical resection. For each observer, the areas under the receiver operating characteristic (ROC) curve were 0.971, 0.959, and 0.967 for MRI and 0.947, 0.950, and 0.943 for CT. The sensitivity differences in positive and negative predictive values between the 2 techniques for each observer were not statistically significant $(P>0.05)$. Gadoxetic acidenhanced MRI and triple-phase MDCT had similar diagnostic performance in the preoperative detection of HCC, but MRI may be better than MDCT in the detection of HCC with a diameter $\leq 1 \mathrm{~cm}$ or according to a subgroup analysis.

Kim et al ${ }^{77}$ compared the diagnostic performance of gadoxetic acid-enhanced MRI with ferucarbotran-enhanced MRI for the detection of liver metastases in 36 of 80 patients with liver metastases. There was a trend toward increased areas under the ROC curve for the gadoxetic acid set (0.950 and 0.948 ) as compared with the ferucarbotran set (0.941 and 0.939) of images, but no significant difference was found for both observers. Sensitivities of the gadoxetic acid set 
(93.8\% and $92.5 \%)$ were also slightly better than those of the ferucarbotran set $(88.8 \%$ and $87.5 \%)$ with no significant difference $(P=0.13)$. The 2 image sets showed similar positive predictive values (98.7\% and 98.6\%, respectively). Gadoxetic acid-enhanced MRI showed comparable diagnostic performance to ferucarbotran-enhanced MRI for the detection of liver metastases.

\section{Summary}

Gadoxetic acid, as a bolus injectable paramagnetic hepatobiliary contrast agent combines features of extracellular agents, such as initial tumor perfusion and enhancement on delayed images of tumors with a large blood pool or with hepatocytes maintaining cell membrane function. The detection and characterization of focal liver disease are significantly improved compared with unenhanced MRI, MRI with unspecific contrast agents, and CT. ${ }^{78}$ Innovative rapid acquisition techniques with near isotropic $3 \mathrm{D}$ pulse sequences with fat saturation parallel the technical progress made by MDCT combined with an impressive improvement in tumor-liver contrast.

\section{Disclosure}

The authors report no conflicts of interest in this work.

\section{References}

1. Livraghi T, Meloni F, Morabito A, Vettori C. Multimodal image-guided tailored therapy of early and intermediate hepatocellular carcinoma: long-term survival in the experience of a single radiologic referral center. Liver Transpl. 2004;10(2 Suppl 1):S98-S106.

2. Thomas MB, Abbruzzese JL. Opportunities for targeted therapies in hepatocellular carcinoma. J Clin Oncol. 2005;23(31):8093-8108.

3. Fargion S, Fracanzani AL, Valenti L. Treatment choices for people infected with HCV. J Antimicrob Chemother. 2004;53(5):708-712.

4. Bruno S, Crosignani A, Maisonneuve P, Rossi S, Silini E, Mondelli MU. Hepatitis $C$ virus genotype $1 \mathrm{~b}$ as a major risk factor associated with hepatocellular carcinoma in patients with cirrhosis: a seventeen-year prospective cohort study. Hepatology. 2007;46(5):1350-1356.

5. El-Serag HB. Hepatocellular carcinoma: an epidemiologic view. JClin Gastroenterol. 2002;35(5 Suppl 2):S72-S78.

6. El-Serag HB. Hepatocellular carcinoma and hepatitis $\mathrm{C}$ in the United States. Hepatology. 2002;36(5 Suppl 1):S74-S83.

7. El-Serag HB. Hepatocellular carcinoma: recent trends in the United States. Gastroenterology. 2004;127(5 Suppl 1):S27-S34.

8. Szymanska K, Chen JG, Cui Y, et al. TP53 R249S mutations, exposure to aflatoxin, and occurrence of hepatocellular carcinoma in a cohort of chronic hepatitis B virus carriers from Qidong, China. Cancer Epidemiol Biomarkers Prev. 2009;18(5):1638-1643.

9. http://www.usclivercancer.org/.

10. Vivarelli M, Bellusci R, Cucchetti A, et al. Low recurrence rate of hepatocellular carcinoma after liver transplantation: better patient selection or lower immunosuppression? Transplantation. 2002;74(12): 1746-1751.

11. El-Serag HB, Marrero JA, Rudolph L, Reddy KR. Diagnosis and treatment of hepatocellular carcinoma. Gastroenterology. 2008;134(6): $1752-1763$.
12. El-Serag HB, Rudolph KL. Hepatocellular carcinoma: epidemiology and molecular carcinogenesis. Gastroenterology. 2007;132(7): 2557-2576.

13. http://search.medscape.com/emedicine-search?queryText=liver\% 20tumor.

14. Shen YH, Fan J, Wu ZQ, et al. Focal nodular hyperplasia of the liver in 86 patients. Hepatobiliary Pancreat Dis Int. 2007;6(1):52-57.

15. Faria SC, Iyer RB, Rashid A, Whitman GJ. Hepatic adenoma. AJR Am J Roentgenol. 2004;182(6):1520.

16. Kasper HU, Drebber U, Dries V, Dienes HP. [Liver metastases: incidence and histogenesis]. Z Gastroenterol. 2005;43(10):1149-1157.

17. Kanematsu M, Kondo H, Goshima S, et al. Imaging liver metastases: review and update. Eur J Radiol. 2006;58(2):217-228.

18. Weinmann HJ, Schuhmann-Giampieri G, Schmitt-Willich H, Vogler $\mathrm{H}$, Frenzel T, Gries H. A new lipophilic gadolinium chelate as a tissuespecific contrast medium for MRI. Magn Reson Med. 1991;22(2): 233-237; discussion 242.

19. Cavagna F, Dapra M, Maggioni F, de Haen C, Felder E. Gd-BOPTA/ Dimeg: experimental disease imaging. Magn Reson Med. 1991;22(2): 329-333; discussion 43-46.

20. Young SW, Bradley B, Muller HH, Rubin DL. Detection of hepatic malignancies using Mn-DPDP (manganese dipyridoxal diphosphate) hepatobiliary MRI contrast agent. Magn Reson Imaging. 1990; 8(3):267-276

21. Runge VM, Pels Rijcken TH, Davidoff A, Wells JW, Stark DD. Contrast-enhanced MR imaging of the liver. J Magn Reson Imaging. 1994;4(3):281-289.

22. Mitchell DG. Hepatobiliary contrast material: a magic bullet for sensitivity and specificity? Radiology. 1993;188(1):21-22.

23. Pascolo L, Cupelli F, Anelli PL, et al. Molecular mechanisms for the hepatic uptake of magnetic resonance imaging contrast agents. Biochem Biophys Res Commun. 1999;257(3):746-752.

24. Schuhmann-Giampieri G, Frenzel T, Schmitt-Willich H. Pharmacokinetics in rats, dogs and monkeys of a gadolinium chelate used as a liver-specific contrast agent for magnetic resonance imaging. Arzneimittelforschung. 1993;43(8):927-931.

25. Schuhmann-Giampieri G, Schmitt-Willich H, Frenzel T, Schitt-Willich H. Biliary excretion and pharmacokinetics of a gadolinium chelate used as a liver-specific contrast agent for magnetic resonance imaging in the rat. J Pharm Sci. 1993;82(8):799-803.

26. Schuhmann-Giampieri G, Schmitt-Willich H, Press WR, Negishi C, Weinmann HJ, Speck U. Preclinical evaluation of Gd-EOB-DTPA as a contrast agent in MR imaging of the hepatobiliary system. Radiology. 1992;183(1):59-64.

27. Hamm B, Staks T, Muhler A, et al. Phase I clinical evaluation of Gd-EOB-DTPA as a hepatobiliary MR contrast agent: safety, pharmacokinetics, and MR imaging. Radiology. 1995;195(3):785-792.

28. Muhler A, Clement O, Saeed M, et al. Gadolinium-ethoxybenzyl-DTPA, a new liver-directed magnetic resonance contrast agent. Absence of acute hepatotoxic, cardiovascular, or immunogenic effects. Invest Radiol. 1993;28(1):26-32.

29. Muhler A, Clement O, Vexler V, Berthezene Y, Rosenau W, Brasch RC. Hepatobiliary enhancement with Gd-EOB-DTPA: comparison of spinecho and STIR imaging for detection of experimental liver metastases. Radiology. 1992;184(1):207-213.

30. Muhler A, Heinzelmann I, Weinmann HJ. Elimination of gadoliniumethoxybenzyl-DTPA in a rat model of severely impaired liver and kidney excretory function. An experimental study in rats. Invest Radiol. 1994;29(2):213-6.

31. Muhler A, Weinmann HJ. Biodistribution and excretion of $153 \mathrm{Gd}-$ labeled gadolinium ethoxybenzyl diethylenetriamine pentaacetic acid following repeated intravenous administration to rats. Acad Radiol. 1995;2(4):313-318.

32. Schmitt-Willich H, Brehm M, Ewers CL, et al. Synthesis and physicochemical characterization of a new gadolinium chelate: the liver-specific magnetic resonance imaging contrast agent Gd-EOB-DTPA. Inorg Chem. 1999;38(6):1134-1144. 
33. Schuhmann-Giampieri G. Nonlinear pharmacokinetic modeling of a gadolinium chelate used as a liver-specific contrast agent for magnetic resonance imaging. Arzneimittelforschung. 1993;43(9):1020-1024.

34. Schuhmann-Giampieri G. Liver contrast media for magnetic resonance imaging. Interrelations between pharmacokinetics and imaging. Invest Radiol. 1993;28(8):753-761.

35. Schuhmann-Giampieri G, Mahler M, Roll G, Maibauer R, Schmitz S. Pharmacokinetics of the liver-specific contrast agent Gd-EOB-DTPA in relation to contrast-enhanced liver imaging in humans. J Clin Pharmacol. 1997;37(7):587-596.

36. Bollow M, Taupitz M, Hamm B, Staks T, Wolf KJ, Weinmann HJ. Gadolinium-ethoxybenzyl-DTPA as a hepatobiliary contrast agent for use in MR cholangiography: results of an in vivo phase-I clinical evaluation. Eur Radiol. 1997;7(1):126-132.

37. Reimer P, Rummeny EJ, Shamsi K, et al. Phase II clinical evaluation of Gd-EOB-DTPA: dose, safety aspects, and pulse sequence. Radiology. 1996;199(1):177-183.

38. Hammerstingl R, Zangos S, Schwarz W, et al. Contrast-enhanced MRI of focal liver tumors using a hepatobiliary MR contrast agent: detection and differential diagnosis using Gd-EOB-DTPA-enhanced versus Gd-DTPA-enhanced MRI in the same patient. Acad Radiol. 2002; 9 Suppl 1:S119-S120.

39. Zangos S, Hammerstingl R, Mack MG, et al. Renal enhancement and excretion of the hepatobiliary contrast agent Gd-EOB-DTPA. Rofo. 2001;173(11):1034-1040.

40. Vogl TJ, Kummel S, Hammerstingl R, et al. Liver tumors: comparison of MR imaging with Gd-EOB-DTPA and Gd-DTPA. Radiology. 1996;200(1):59-67.

41. Hammerstingl R, Huppertz A, Breuer J, et al. Diagnostic efficacy of gadoxetic acid (Primovist)-enhanced MRI and spiral CT for a therapeutic strategy: comparison with intraoperative and histopathologic findings in focal liver lesions. Eur Radiol. 2008;18(3):457-467.

42. Huppertz A, Balzer T, Blakeborough A, et al. Improved detection of focal liver lesions at MR imaging: multicenter comparison of gadoxetic acid-enhanced MR images with intraoperative findings. Radiology. 2004;230(1):266-275.

43. Bluemke DA, Sahani D, Amendola M, et al. Efficacy and safety of MR imaging with liver-specific contrast agent: U.S. multicenter phase III study. Radiology. 2005;237(1):89-98.

44. http://www.medicalnewstoday.com/articles/114315.php.

45. http://www.clinicaltrial.gov/ct2/show/NCT00924248.

46. http://clinicaltrials.gov/ct2/show/NCT00526188.

47. Breuer J. Study of the Safety of Gd-EOB-DTPA and the Effect of Hepatic and Renal Impairment on the Pharmacokinetics (PK) and the Pharmacodynamics (PD) of Gd-EOB-DTPA after a Single Intravenous Dose in Volunteer Patients, B. Berlin, Germany: Schering AG; 2003.

48. Zech CJ, Grazioli L, Jonas E, et al. Health-economic evaluation of three imaging strategies in patients with suspected colorectal liver metastases: Gd-EOB-DTPA-enhanced MRI vs. extracellular contrast media-enhanced MRI and 3-phase MDCT in Germany, Italy and Sweden. Eur Radiol. 2009;19 Suppl 3:S753-S763.

49. Zech CJ, Herrmann KA, Reiser MF, Schoenberg SO. MR imaging in patients with suspected liver metastases: value of liver-specific contrast agent Gd-EOB-DTPA. Magn Reson Med Sci. 2007;6(1):43-52.

50. Kuhn JP, Hegenscheid K, Siegmund W, Froehlich CP, Hosten N, Puls R. Normal dynamic MRI enhancement patterns of the upper abdominal organs: gadoxetic acid compared with gadobutrol. AJR Am J Roentgenol. 2009;193(5):1318-1323.

51. Kim YK, Kwak HS, Kim CS, Han YM. Detection and characterization of focal hepatic tumors: a comparison of T2-weighted MR images before and after the administration of gadoxectic acid. J Magn Reson Imaging. 2009;30(2):437-443.

52. Reimer P, Rummeny EJ, Daldrup HE, et al. Enhancement characteristics of liver metastases, hepatocellular carcinomas, and hemangiomas with Gd-EOB-DTPA: preliminary results with dynamic MR imaging. Eur Radiol. 1997;7(2):275-280.
53. Huppertz A, Haraida S, Kraus A, et al. Enhancement of focal liver lesions at gadoxetic acid-enhanced MR imaging: correlation with histopathologic findings and spiral CT-initial observations. Radiology. $2005 ; 234(2): 468-478$

54. Saito K, Kotake F, Ito N, et al. Gd-EOB-DTPA enhanced MRI for hepatocellular carcinoma: quantitative evaluation of tumor enhancement in hepatobiliary phase. Magn Reson Med Sci. 2005;4(1):1-9.

55. Shimofusa R, Ueda T, Kishimoto T, et al. Magnetic resonance imaging of hepatocellular carcinoma: a pictorial review of novel insights into pathophysiological features revealed by magnetic resonance imaging. J Hepatobiliary Pancreat Surg. Epub 2009 Oct 7.

56. Ba-Ssalamah A, Fakhrai N, Matzek WK, et al. Magnetic resonance imaging of liver malignancies. Top Magn Reson Imaging. 2007;18(6): 445-455.

57. Fujita M, Yamamoto R, Takahashi M, et al. Paradoxic uptake of Gd-EOB-DTPA by hepatocellular carcinoma in mice: quantitative image analysis. J Magn Reson Imaging. 1997;7(4):768-770.

58. Jung G, Breuer J, Poll LW, et al. Imaging characteristics of hepatocellular carcinoma using the hepatobiliary contrast agent Gd-EOB-DTPA Acta Radiol. 2006;47(1):15-23.

59. Sun HY, Lee JM, Shin CI, et al. Gadoxetic acid-enhanced magnetic resonance imaging for differentiating small hepatocellular carcinomas ( $<$ or $=2 \mathrm{~cm}$ in diameter) from arterial enhancing pseudolesions: special emphasis on hepatobiliary phase imaging. Invest Radiol. 2010;45(2):96-103.

60. Narita M, Hatano E, Arizono S, et al. Expression of OATP1B3 determines uptake of Gd-EOB-DTPA in hepatocellular carcinoma. $J$ Gastroenterol. 2009;44(7):793-798.

61. Frericks BB, Loddenkemper C, Huppertz A, et al. Qualitative and quantitative evaluation of hepatocellular carcinoma and cirrhotic liver enhancement using Gd-EOB-DTPA. AJR Am J Roentgenol. 2009; 193(4):1053-1060.

62. Kim SH, Kim SH, Lee J, et al. Gadoxetic acid-enhanced MRI versus triple-phase MDCT for the preoperative detection of hepatocellular carcinoma. AJR Am J Roentgenol. 2009;192(6):1675-1681.

63. Ba-Ssalamah A, Uffmann M, Saini S, Bastati N, Herold C, Schima W. Clinical value of MRI liver-specific contrast agents: a tailored examination for a confident non-invasive diagnosis of focal liver lesions. Eur Radiol. 2009;19(2):342-357.

64. Brody JM, Schafer L, Tung GA, Breuer J, Shamsi K. Conspicuity of liver hemangiomas: short tau inversion recovery, T1, and T2 imaging with gadolinium ethoxybenzyl diethylenetriaminepentaacetic acid. J Magn Reson Imaging. 2005;21(4):391-397.

65. Zech CJ, Grazioli L, Breuer J, Reiser MF, Schoenberg SO. Diagnostic performance and description of morphological features of focal nodular hyperplasia in Gd-EOB-DTPA-enhanced liver magnetic resonance imaging: results of a multicenter trial. Invest Radiol. 2008;43(7): 504-511.

66. Kacl GM, Hagspiel KD, Marincek B. Focal nodular hyperplasia of the liver: serial MRI with Gd-DOTA, superparamagnetic iron oxide, and Gd-EOB-DTPA. Abdom Imaging. 1997;22(3):264-267.

67. Giovanoli O, Heim M, Terracciano L, Bongartz G, Ledermann HP. MRI of hepatic adenomatosis: initial observations with gadoxetic acid contrast agent in three patients. AJR Am J Roentgenol. 2008;190(5) W290-W293.

68. Kuwatsuru R. [MR imaging of liver metastasis using Gd-EOB-DTPA]. Gan To Kagaku Ryoho. 2009;36(8):1234-1241.

69. Shimada M, Hayashi S, Senoo A, et al. [Dynamic MR hepatocholangiography with the SIP Fast GRE (saturation inversion projection fast gradient echo) method]. Nippon Igaku Hoshasen Gakkai Zasshi. 1997;57(11):681-683.

70. Shimizu J, Dono K, Gotoh M, et al. Evaluation of regional liver function by gadolinium-EOB-DTPA-enhanced MR imaging. Dig Dis Sci. 1999;44(7):1330-1337.

71. Shuter B, Wang SC, Roche J, Briggs G, Pope JM. Relaxivity of GdEOB-DTPA in the normal and biliary obstructed guinea pig. J Magn Reson Imaging. 1998;8(4):853-861. 
72. Tschirch FT, Struwe A, Petrowsky H, Kakales I, Marincek B, Weishaupt D. Contrast-enhanced MR cholangiography with Gd-EOB-DTPA in patients with liver cirrhosis: visualization of the biliary ducts in comparison with patients with normal liver parenchyma. Eur Radiol. 2008;18(8):1577-1586.

73. Carlos RC, Branam JD, Dong Q, Hussain HK, Francis IR. Biliary imaging with Gd-EOB-DTPA: is a 20-minute delay sufficient? Acad Radiol. 2002;9(11):1322-1325.

74. Carlos RC, Hussain HK, Song JH, Francis IR. Gadolinium-ethoxybenzyl-diethylenetriamine pentaacetic acid as an intrabiliary contrast agent: preliminary assessment. AJR Am J Roentgenol. 2002;179(1):87-92.

75. Filippone A, Blakeborough A, Breuer J, et al. Enhancement of liver parenchyma after injection of hepatocyte-specific MRI contrast media: a comparison of gadoxetic acid and gadobenate dimeglumine. J Magn Reson Imaging. 2010;31(2):356-364.
76. Ichikawa T, Saito K, Yoshioka N, et al. Detection and characterization of focal liver lesions: a Japanese phase III, multicenter comparison between gadoxetic acid disodium-enhanced magnetic resonance imaging and contrast-enhanced computed tomography predominantly in patients with hepatocellular carcinoma and chronic liver disease. Invest Radiol. 2010;45(3):133-141.

77. Kim YK, Lee YH, Kwak HS, Kim CS, Han YM. Detection of liver metastases: gadoxetic acid-enhanced three-dimensional MR imaging versus ferucarbotran-enhanced MR imaging. Eur J Radiol. 2008;73(1): 131-136.

78. Halavaara J, Breuer J, Ayuso C, et al. Liver tumor characterization: comparison between liver-specific gadoxetic acid disodium-enhanced MRI and biphasic CT - a multicenter trial. J Comput Assist Tomogr. 2006;30(3):345-354
Biologics: Targets \& Therapy

\section{Publish your work in this journal}

Biologics: Targets \& Therapy is an international, peer-reviewed journal focusing on the patho-physiological rationale for and clinical application of Biologic agents in the management of autoimmune diseases, cancers or other pathologies where a molecular target can be identified. This journal is indexed on PubMed Central, CAS, EMBase, Scopus

Submit your manuscript here: http://www.dovepress.com/biologics-targets--therapy-journal

\section{Dovepress}

and the Elsevier Bibliographic databases. The manuscript management system is completely online and includes a very quick and fair peerreview system, which is all easy to use. Visit http://www.dovepress. com/testimonials.php to read real quotes from published authors. 